\title{
Thermal Image Processing for Breast Symmetry Detection Oriented to Automatic Breast Cancer Analysis
}

\author{
Mario I. Chacon-Murguia, Adrian J. Villalobos-Montiel, \\ and Jorge D. Calderon-Contreras \\ Visual Perception Applications on Robotics Lab, Chihuahua Institute of Technology, Mexico \\ mchacon, avillalobos \{ @itchihuahua.edu.mx
}

\begin{abstract}
The present work presents a methodology to automatically detect the symmetry point of breast. In order to achieve this goal, the algorithm corrects thermal image tilt to find a breast symmetry axis, compute a modified symmetric index that can be used as a measure of image quality, breast cosmetic and pathologic issues, and a seed location for a former algorithm reported to automatically achieve breast cancer analysis. The methodology involves filtering, edge detection, windowing edge analysis and shape detection based on the Hough transform. Experimental results show that the proposed method is able to define the symmetry axis as precise as a person do, and correctly detected breast areas in $100 \%$ of the cases considered, allowing automatic breast analysis by a previous algorithm.
\end{abstract}

Keywords: breast segmentation, breast thermography, breast cancer.

\section{Introduction}

Breast thermography is a promising technique for the detection of breast cancer based on infrared technology [1]. A thermographic image shows the temperatures detected in the environment. In the case of breast thermography, temperatures of the different zones of the breast are shown. This allows us to detect areas where there might be a tumor due that in cancerous tissues there is an increased blood supply, and therefore an increased temperature of approximately $0.5^{\circ} \mathrm{C}$ with respect to other tissues. Breast thermography also allows the detection of cancer through the study of asymmetry. If there is a large level of breast asymmetry, this may be due to the presence of a tumor that is being developed in one of the breasts, and is generating more breast mass. Different algorithms have been developed to segment and analyze areas of interest in order to detect possible tumors. But some of these are not automatic, and require the aid of a user to guide the program [2]. Other algorithms need the input images to be already segmented in order to process them correctly [3-5]. In some cases, thermal images are not taken properly so the systematic error increases, and the accuracy of the algorithm decreases [6]. An example of this is when the camera or the patient is inclined toward some direction when the image is taken. None of the reviewed algorithms that perform breast thermography analysis takes this into account, especially if it desired to design a mobile system. If this factor would not be considered, the asymmetry index and the internal segmentation of the breasts can be affected. 
In 2007, a study was conducted at the Medical University of Vienna to define for the first time a breast symmetry index (BSI (C)). The BSI can clearly differentiate between a good and a bad cosmetic appearance (BSI > 30\%), and it is also used for clinical studies [7]. This paper presents the development of an algorithm that automatically locates the breast symmetry point. The algorithm provides important image data so that it can be used for the segmentation of the breasts, and help in the detection of tumors. These data are a level of symmetry between the breasts, and the symmetry axis defined by the angle to the $x$ axis of the image, and its position in the $x$ axis once the image inclination has been corrected. The degree of symmetry between the breasts is used to verify the proper functioning of the proposed algorithm, and it can also be used for detection of a possible tumor if this index is very low. Additionally, the algorithm provides the coordinates of a seed in the image that later can be used to perform internal breast segmentation and therefore determine risk areas.

In [2] a processing algorithm of thermal images that makes the segmentation and analysis of the breasts in order to evaluate the heat patterns in the breast tissue for diagnosis of breast cancer is described. The algorithm has a good performance achieving correct segmentations. However, this algorithm has the disadvantage that it requires an experienced person to indicate a seed, or select the coordinate of the midpoint between the breasts, according to his point of view. This point serves as a starting point for breast detection, so the results of the algorithm varied greatly if another coordinate was used. Therefore, the algorithm proposed in this article is oriented to locate the breast areas in the images, and then determine the coordinates of the seed so that the algorithm mentioned in [2] can work automatically.

The contributions of this paper are; a method to correct image tilt, a modified symmetric index that can be used as a measure of image quality, cosmetic and pathologic issues, and seed location for the algorithm reported in [2] to work automatically on breast cancer analysis. The organization of the paper is structured as follows. Section 2 explains the proposed method. Experimental results are presented in Section 3, and the conclusions are commented in section 4.

\section{Development and Description of the Algorithm}

The algorithm works with thermal images size of $120 \times 160$ pixels, $I(x, y)$. The images used for the design and test of the algorithm correspond to 12 woman, from whom 36 thermal images with a $0.1{ }^{\circ} \mathrm{C}$ resolution were acquired. The image acquisition protocol used and the information of the camera are described in [2]. Since the images were not taken under ideal conditions $[6,8]$, some are clear and other blurred. There was a variety of breast sizes and positions. Based on the variety of images, five of these images that were considered as representative of the others, were chosen for the design stage of the algorithm. The first process performed by the algorithm is to copy the original image and paste it in the center of a blank image, $I_{N}(x, y)$, that has the dimensions of the original image plus 60 pixels on both dimensions. This process allows greater working space when detecting the symmetry axis, and it is very helpful for cases when the position of the person in the image is shifted to any direction compared to the ideal position where the breasts appear at the center of the thermographic image. Then, a Gaussian smoothing filter, is applied to the image in order to reduce the noise 


$$
G(x, y)=I_{N}(x, y) \Rightarrow I_{S}(x, y)
$$

where

$$
G(x, y)=\frac{1}{2 \pi \sigma^{2}} e^{\frac{-\left(x^{2}+y^{2}\right)}{2 \sigma^{2}}}
$$

The $\sigma$ used was 0.5 , as this value preserves the desired extracted edges.

Once the image has been softened, an edge detector is applied. In order to choose the best edge detector, a study was conducted with different edge detector operators such as Sobel, Canny and Prewitt. Figure 1 shows an example of comparison of the edge operators. The Canny operator was selected with a threshold of 0.2 as this allows the detection of the desired breasts and bodies edges in most of the images.

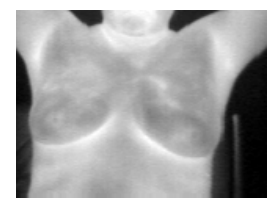

a)

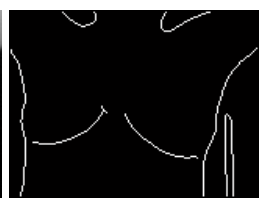

b)

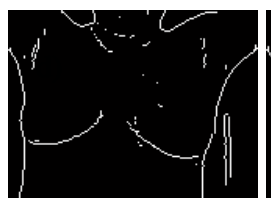

c)

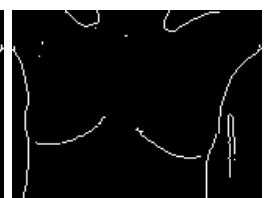

d)

Fig. 1. Edge detection operators: a) Original Image, b) Canny with threshold $=0.2$, c) Sobel with threshold $=0.04, \mathrm{~d}$ ) Prewitt with threshold $=0.05$

The next step is edge dilation in vertical, horizontal and diagonal directions, in order to enhance edges and to have an increased ability to detect symmetry. The dilation is achieved over each edge pixel $p_{e}(x, y)$

$$
\begin{aligned}
& \text { for each } p_{e}(x, y) \\
& \qquad N_{s}\left(p_{e}(x, y)\right)=1 .
\end{aligned}
$$

After detecting the edges of the breasts, the image must be rotated. This is very important if it is desired to segment the breasts in two different regions. Otherwise, some pixels from one breast would be considered as pixels of the other breast and it can lead to different results when the internal analysis is performed. The rotation operator is defined by

$$
\begin{aligned}
& x_{2}=\cos (\theta)\left(x_{1}-x_{0}\right)-\sin (\theta)\left(y_{1}-y_{0}\right)+x_{0} \\
& y_{2}=\sin (\theta)\left(x_{1}-x_{0}\right)+\cos (\theta)\left(y_{1}-y_{0}\right)+y_{0}
\end{aligned}
$$

where $\theta$ is the angle of rotation in a clockwise direction and $\left(x_{0}, y_{0}\right)$ are the coordinates of the center of rotation [9]. The developed algorithm considers $\left(x_{0}, y_{0}\right)$ as the center point of the image. It is important to note that if an image is rotated its dimensions change.

To carry out the angular correction and the symmetry analysis of the thermographic images, the algorithm looks in the image for those conditions in which an axis indicates a higher degree of symmetry. By reviewing the position of the breasts in the images used for the design of the algorithm, a common area in which it is very possible to find the breasts in any other similar image was delimited. This area was named the test area and it is defined as the region where $y$ is in the range [30,151]. Two adjoining windows of size of $70 \times 121$ pixels, $W_{L}$ and $W_{R}$, are defined inside the test 
area located in the left margin and at row 151. The specified parameters should be adapted in order for the algorithm to process images from other data bases. $W_{L}$ and $W_{R}$ are horizontal and continuously shifted and at each position, then $W_{R}$ is horizontally reflected to compute a Boolean AND operation with $W_{L}$. The resulting image has pixels with a value of 1 , only when $W_{L}$ and the reflected $W_{R}$ have both pixels of value 1 at the same pixel position. An example of this operation is shown in Figure 2.

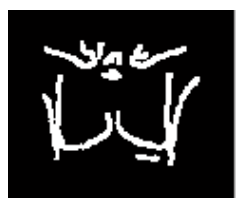

a)

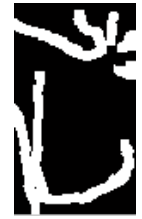

b)

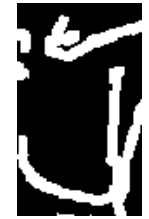

c)

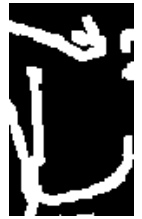

d)

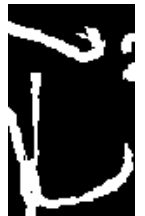

e)

Fig. 2. a) Edges image, b) $W_{L}$, c) $W_{R}$, d) $W_{R}$ reflected, e) Result of the AND operation between $W_{L}$ and $W_{R}$ reflected

The symmetry index, $S I$, is then computed by counting the pixels that resulted with a value of 1 from this operator. $S I$ is then used to obtain the symmetry axis, $S A$, as follows

$$
\begin{aligned}
& S I_{M}=0 \\
& \text { for orientation }-10^{\circ} \text { to } 10^{\circ} \text { and increments of } 1^{\circ} \\
& \{\text { compute } S I \\
& \text { if } S I>S I_{M} \text { THEN } S I_{M}=S I \text { and } S A=\text { orientation } \\
& \text { \} }
\end{aligned}
$$

The algorithm detects the angle and windows shift position that defines a symmetry axis where there is the greatest symmetry between the breasts and surrounding areas. Since it doesn't exist a standardized way to measure symmetry, the count of $1 \mathrm{~s}$ from the best condition was defined as the symmetry level of an image. Figure 3 shows an example in which the symmetry axis was found for a breast thermographic image. In this example, the symmetry axis is generated when the image is rotated $5^{\circ}$ and at a position of 111 in the horizontal axis. The symmetry level in this image was determined to be 1151, which was basically determined by the information generated by the AND operation. Figure 4 shows the results displayed by the algorithm once an image has been processed. It displays the main steps to determine the symmetry axis, such as the best condition obtained after using the AND operator, and the symmetry axis over the borders image and over the original image.

Once the algorithm determines the degree of symmetry, it analyzes whether it is within an adequate range. For example, it is helpful to detect if the input image presents problems in its acquisition, or if there is great asymmetry between the breasts perhaps due to a malformation or a possible tumor. If the symmetry index is less than 700 , the algorithm sends an error message related to image quality or a pathological issue. This threshold was selected because it was observed that good quality and nonpathologic images showed symmetry levels above this value.

Other important result of the proposed algorithm is that it also selects a coordinate in order to be used in [2] as the seed to perform automatic breast cancer analysis. Once the image angle has been corrected, the position of the axis will provide the $x$ 


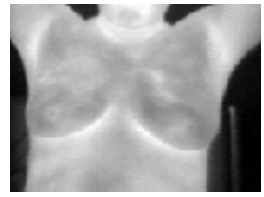

a)

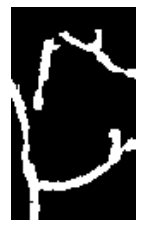

b)

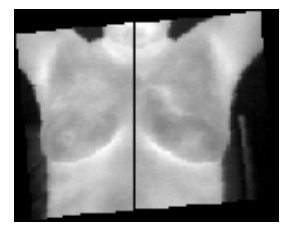

c)

Fig. 3. Determination of symmetry axis: a) Original Image b) AND image generated with the greatest symmetry level conditions, c) Rotation of the image and positioning of the symmetry axis

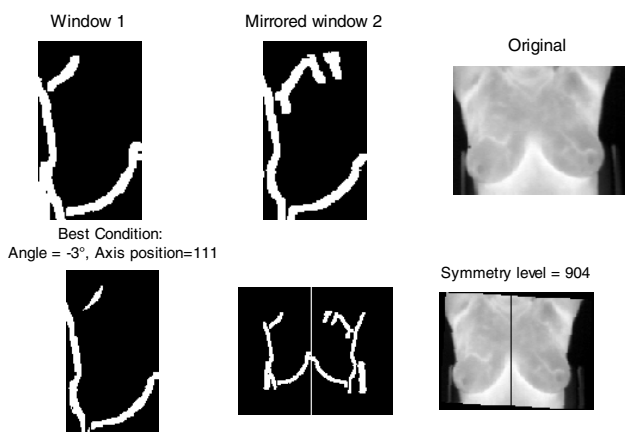

Fig. 4. Results displayed of the algorithm

component of the location of the seed. In order to find the $y$ component, the Hough transform is used. Since breasts have similar shapes to circles, detecting circles from a breast thermography is helpful to locate the breasts in an image. The equation of a circle is given by

$$
\left(x-x_{r}\right)^{2}+\left(y-y_{r}\right)^{2}=S^{2}
$$

With this equation and the aid of the Hough transform, the algorithm locates 2 circles where the breasts are most likely positioned as shown in Figure 5. Because the formula of the circle has three parameters $(x r, y r, S)$ [10], the Hough transform should be performed in a three dimensional space, where each pixel $\left(x_{i}, y_{j}\right)$ is mapped to an accumulator $\mathrm{H}(x r, y r, S)$ in the Hough space. Each point that satisfies the Equation (6) will cause the accumulator increase one. The circles are defined by finding the accumulators with maximum values [11].
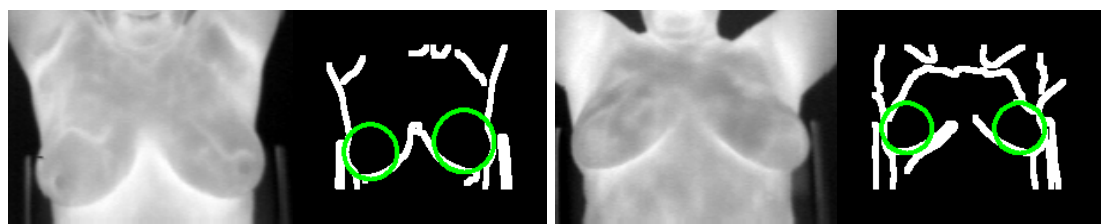

Fig. 5. Use of the Hough transform for detection of circles where the breasts might be located

One of the conditions given to the algorithm is that the radius of possible circles must be according to possible breast sizes and breast separation to avoid false 
detection and computational burden. The average of the $y$ components of the circles' center is used as the $y$ component of the seed only if the vertical distance between both origins is equal or less than 20 pixels. If this is not true, the $y$ component of the circles closer to 110 , the ideal position determined by inspection of the thermal images, will be considered as the $y$ component for the seed. This situation indicates that one of the circles was not correctly detected, and an assumption that at least one of the circles detected belong to a breast is made. Figure 6 shows examples of the seeds selected for 3 different thermographic images. In Figures $6 a$ and $6 \mathrm{~b}$, both breasts positions were detected correctly using the Hough transform. But in Figure 6c, only one breast was detected correctly. Therefore, the $y$ component of the seed is based only on the circle that correctly detected a breast position. As explained in the next section, when the algorithm was tested with all the images available, in $100 \%$ of the cases the algorithm was able to detect correctly at least of one of the breasts, therefore the seed for the algorithm of [2] is correctly determined.

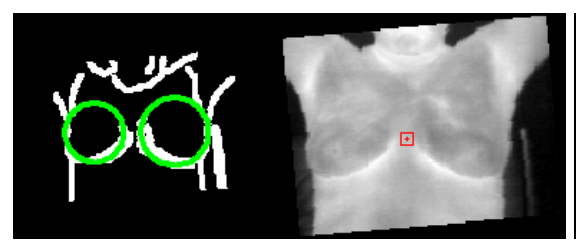

a)

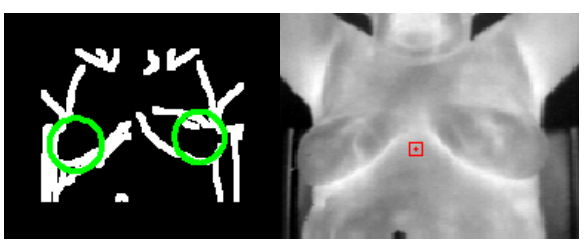

b)

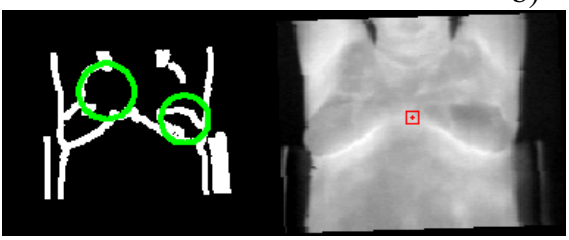

c)

Fig. 6. Examples of seed position selection based on the circles detected

\section{Experimental Results}

To evaluate the performance of the algorithm, it was tested with 36 different breast thermographies that were available. Some of these showed very poor quality. The performance of the algorithm about correctly determining the symmetry axis was first qualitatively evaluated for each of the processed images and classified as very good $(\mathrm{VG})$, good (G) and bad (B). The criterion used was based on the location of the symmetry axis, and the body parts shown after the AND operator. VG performance involves correctly placing the symmetry axis and having few parts that do not belong to the breasts in the AND image. G performance involves correctly placing the symmetry axis, but having several parts in the AND image that don't belong to the breast areas. B performance involves a wrong location of the symmetry axis or many noise or additional parts shown in the AND image.

Of all the processed images, in 3 of them the algorithm was classified as B, in 12 images it was $\mathrm{G}$ and in the remaining 21, the performance was VG. At first glance, it was observed that the algorithm was doing an excellent job determining the position of the axis of symmetry. Table 1 contains some of the results provided by the algorithm and 
the performance evaluation for only 13 representative images due to space restrictions. As it can observed, the angle corrections ranged $\left[-3^{\circ}, 5^{\circ}\right]$, so the algorithm considers about twice angle ranging which could be reduced to avoid unnecessary processing.

Table 1. Results of the algorithm for 13 representative images

\begin{tabular}{|c|c|c|c|c|}
\hline Image & $\begin{array}{c}\text { Correction } \\
\text { Angle }\end{array}$ & $\begin{array}{c}\text { Axis } \\
\text { Position }\end{array}$ & $\begin{array}{c}\text { Symmetry } \\
\text { level }\end{array}$ & Performance \\
\hline 1 & 0 & 108 & 724 & G \\
\hline 2 & -3 & 110 & 1497 & G \\
\hline 3 & -3 & 111 & 1402 & G \\
\hline 4 & 0 & 104 & 912 & G \\
\hline 5 & -4 & 119 & 911 & VG \\
\hline 6 & -3 & 112 & 704 & VG \\
\hline 7 & -2 & 112 & 1198 & VG \\
\hline 8 & 0 & 104 & 2750 & B \\
\hline 9 & 0 & 95 & 1531 & VG \\
\hline 10 & -3 & 111 & 904 & VG \\
\hline 11 & 0 & 104 & 1333 & VG \\
\hline 12 & 5 & 111 & 1151 & VG \\
\hline 13 & 5 & 109 & 1252 & VG \\
\hline
\end{tabular}

Reviewing the obtained positions of the axes of symmetry in the $x$ axis, it was found that they were very different. Likewise, the symmetry level values obtained varied from 724 to 2750 , and symmetry levels had an average of 1198 considering all the images. Figure 7 shows the positions of the detected symmetry axis for 2 images that were not used during the design of the algorithm. Although in both cases the symmetry axes were placed correctly, case (a) was considered G, and not VG as in case (b), because the breast curve did not appeared on its corresponding AND image. The border of the body was helpful in this case because it facilitated to find the symmetry of the whole image. If the body curves had been deleted, as presented previously as an option, then it would had been harder to find the symmetry axis. The corresponding AND images of the thermographies in Figure 7 are shown in Figure 8. In both cases, it can be observed that the chair in which patients were sitting is appearing in the AND images, and therefore, the algorithm is taking it into account for the symmetry detection, introducing the possibility of errors. The used images to develop and test the algorithm were provided by a third person. But for future uses, it is recommended to sit the patients in a bench instead of a chair, and control other similar conditions at the time of the acquisition to avoid this kind of problems.

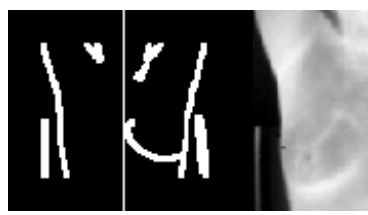

a)

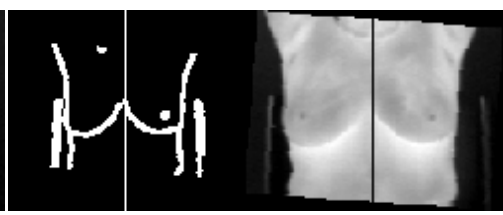

b)

Fig. 7. Symmetry axes found with levels a) 912 and b) 755 


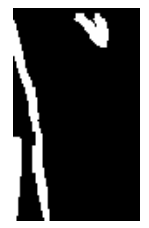

a)

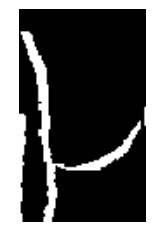

b)

Fig. 8. AND images obtained from the thermographies from Figure 7

Figure 9 shows the case of an image not considered for the algorithm design and evaluation, but it is used to exemplify the importance of the symmetry average value. The algorithm performance was classified as B, this was due mainly because the image was taken under undesired conditions. The breast area temperature of the patient was not stabilized at the time of the image acquisition, so the image showed high variation on this area generating many edges which caused that the defined symmetry axis would not be on the desired position.

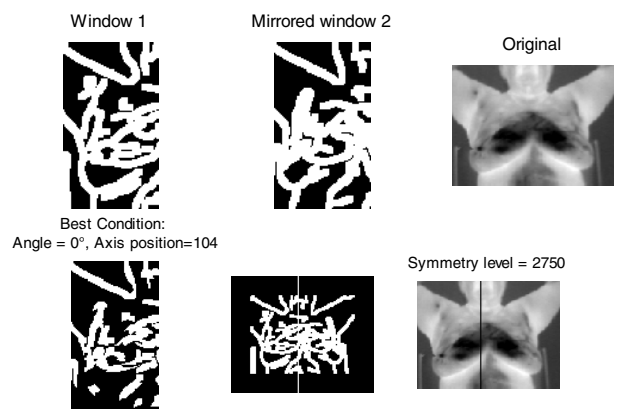

Fig. 9. Image in which the algorithm was classified as having a bad performance

This image resulted with a high symmetry level of 2750 . Therefore, it can be noticed that a higher symmetry level does not necessarily mean that a better symmetry axis was detected. Better symmetry axis detection, as defined for this algorithm, is given when the symmetry level is closer to 1123 , which is the average symmetry level obtained of images in which the program performance was VG. Having symmetry levels far away from this average may indicate, as in this example, the presence of low quality thermographic images to be processed by the algorithm. This situation also happened with the other images in which the algorithm performance was considered as B.

Another case using an image not considered for the algorithm design and evaluation to illustrate the robustness of the proposed algorithm is illustrated in Figure 10. This figure shows how a blurry image was correctly processed by the algorithm. Although this image was not taken properly, the symmetry axis was placed correctly due to the symmetry in the fuzziness of the image. The symmetry level of this image was 704, which is a value far from the ideal symmetry level and very close to the threshold that sends an error message to the user. 

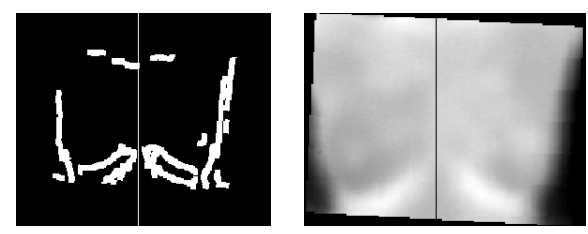

Fig. 10. Correct image processing of a blurry image

In order to evaluate the performance of the algorithm quantitatively, 10 people with no previous medical image analysis and interpretation training were provided with 5 digital breast thermographic images. They had to draw digitally on the images a thin red line in the axis of symmetry of the breasts according to their point of view. Their images were processed in order to calculate the angle specified by each person. The obtained angle values are in Table 2 to show how the values were spread out, and they were compared with those calculated by the algorithm, which are also shown in the table. The fields in yellow are those in which there was a difference equal or less than $1^{\circ}$ with respect to the angles provided by the algorithm (shown in the blue fields). The table also considers the average of the values determined by the 10 people. The average of the 5 images resulted having a difference of $1^{\circ}$ or below when compared with the angle values obtained by the algorithm. Therefore, it was concluded that the algorithm can detect the symmetry axes as well as a real person can do. Taking into account that the angle values obtained from the people varied greatly, the algorithm also has better precision than humans, and consequently can be trusted more than a person.

Table 2. Angles of rotation specified by the algorithm and 10 people

\begin{tabular}{|l|r|r|r|r|r|}
\hline Image & Im1 & Im2 & Im3 & Im4 & Im5 \\
\hline Algorithm & -2 & -3 & 5 & 0 & 0 \\
\hline Person 1 & -6 & -5 & 7 & -2 & -4 \\
\hline Person 2 & -3 & -3 & 3 & 0 & -1 \\
\hline Person 3 & -1 & -2 & 1 & 0 & 0 \\
\hline Person 4 & -4 & -4 & 9 & 4 & -2 \\
\hline Person 5 & -4 & -3 & 6 & -1 & -1 \\
\hline Person 6 & 0 & 0 & 6 & 4 & 2 \\
\hline Person 7 & -3 & -4 & 5 & 5 & 0 \\
\hline Person 8 & -1 & -8 & 6 & -2 & 1 \\
\hline Person 9 & -6 & -5 & 13 & -1 & 0 \\
\hline Person 10 & -2 & -3 & 4 & -2 & -1 \\
\hline Average of people & -3 & -3.7 & 6 & 0.5 & -0.6 \\
\hline
\end{tabular}

The performance of the algorithm according to the seed selection was also evaluated. When the algorithm was tested with the 36 images available, in all of them, at least one of the breasts positions in each image was correctly defined by the Hough circles. In $61.1 \%$ of the images, the algorithm correctly placed the circles over the 2 different breasts. In $19.4 \%$ of the cases, the algorithm placed both circles over only one breast, and in the remaining $19.4 \%$ of the images, only one of the breasts was wrongly located in the image, while the other one was placed correctly. However, in this last case, the 
algorithm successfully used the correct circle to set the $y$ component value of the seed. The selected seed coordinates of the 36 images were considered acceptable taking into account the criteria used in [2], allowing the algorithm in [2] correctly segment automatically the regions of interest and perform cancer diagnosis with the given seeds. The difference that exists in the calculation of the $y$ component of the seed, between having correctly detected one or two circles over the breast area does not affect significantly the acceptance of a seed, but having two circles correctly detected doubles the possibility of setting correctly the $y$ component of the seed coordinate.

\section{Conclusions}

It was possible the design of an algorithm that automatically process breast thermographic images in order to detect the symmetry axis and therefore, correct the rotation error and provide a level of symmetry. The developed algorithm was tested and had an excellent performance. It allowed successful process of images, even if they were of poor quality. Likewise, the algorithm provided in $100 \%$ of the images acceptable seed coordinates to aid another algorithm perform automatic internal breast segmentation.

Acknowledgements. The authors thanks to DGEST, for the support of this research.

\section{References}

1. Sharon, G.H., Budzar, A.U., Hoertobagyi, G.N.: Breast Cancer in Men. Annals of International Medicine 137(8), 678-687 (2002)

2. Calderon, J.D., Chacon, M.I.: Development of a breast tissue segmentation algorithm in thermal images for breast cancer detection. In: Proceedings of the National Congress on University and Health (2012)

3. Etehad Tavakol, M., Sadri, S., Ng, E.Y.K.: Application of K- and fuzzy c-means for color segmentation of thermal infrared breast images. Journal of Medical Systems 34(1), 35-42 (2010)

4. Boquete, L., Ortega, S., Miguel, J.M., Rodríguez, J.M., Blanco, R.: Automated detection of breast cancer in thermal infrared images, Based on independent component analysis. J. Medical Systems 36(1), 103-111 (2012)

5. Kapoor, P., Prasad, S.V.A.V., Patni, S.: Image Segmentation and Asymmetry Analysis of Breast Thermograms for Tumor Detection. International Journal of Computer Applications 50(9), 40-45 (2012)

6. Ammer, K., Ring, F.J.: Standard procedures for infrared imaging in medicine. In: Medical Infrared Imaging, pp. 32.1-32.14. CRC Press, Boca Raton (2012)

7. Fitzal, F., et al.: The use of a breast symmetry index for objective evaluation of breast cosmesis. In: The Breast, vol. 16, pp. 429-435. Elsevier, Edinburgh (2007)

8. Ng, E.Y.K.: A review of thermography as promising non-invasive detection modality for breast tumor. International Journal of Thermal Sciences 48(5), 849-859 (2009)

9. Ballard, D.H., Brown, C.M.: Computer Vision. Prentice Hall, Englewood Cliffs (1982)

10. Ballard, D.H.: Generalizing the Hough transform to detect arbitrary shapes. Pattern Recognition 13(2), 111-122 (1981)

11. Chen, B., Ma, Z.: Automated image segmentation and asymmetry analysis for breast using infrared images. In: International Workshop on Education Technology and Training (2008) 